\title{
Impacto del componente hiperclorémico de la acidosis metabólica en el estado de hidratación y en el tratamiento de la cetoacidosis diabética
}

\author{
Impact of the hyperchloremic component of metabolic acidosis \\ on the patient's hydration status and the treatment of diabetic \\ ketoacidosis
}

\author{
Dr. Ismael Toledo ${ }^{a}$, Dra. Raquel Wainsztein ${ }^{a}$, Dra. Carla Mannuccib, \\ Dra. Mabel Ferraro ${ }^{b}$, Dr. Juan Ferreira ${ }^{c}$ y Dr. Alejandro Balestracci ${ }^{a}$
}

\section{RESUMEN}

Introducción. La cetoacidosis diabética (CAD) se caracteriza por acidosis metabólica (AM) con anión restante (AR) elevado, aunque, ocasionalmente, puede presentar hipercloremia. Se postuló que la presencia de hipercloremia inicial podría reflejar un mejor estado de hidratación; sin embargo, su prevalencia y su impacto en el tratamiento de la CAD se desconoce.

Objetivos. Determinar la prevalencia de AMcon componente hiperclorémico previo al inicio del tratamiento y evaluar si su presencia se asocia con mejor estado de hidratación y con menor tiempo de salida de la CAD, en comparación con los pacientes con AR elevado exclusivo.

a. Unidad de Nefrología.

b. Servicio de Nutrición y Diabetes.

c. Comité de Docencia e Investigación.

Hospital General de Niños Pedro de Elizalde, Ciudad Autónoma de Buenos Aires, Argentina.

Correspondencia: Dr. Ismael Toledo: maetoledo77@hotmail. com

Financiamiento:
El presente trabajo
se realizó en el
marco de una Beca
de Investigación del
Departamento de
Pediatría de la Facultad
de Medicina de la
Universidad de Buenos
Aires otorgada al Dr.
Ismael Toledo.

Conflicto de intereses: Ninguno que declarar.

Recibido: 9-5-2017 Aceptado: 3-10-2017
Pacientes y métodos. Se agruparon los pacientes internados con CAD (período entre enero de 2014 y junio de 2016) según presentaran, al ingresar, AM con AR elevado exclusivo o con hipercloremia y se compararon sus variables clínicas, de laboratorio y la respuesta al tratamiento.

Resultados. Se incluyeron 40 pacientes [17 varones, mediana de edad: 14,5 años (2,4-18)], 22 con AM con componente hiperclorémico (prevalencia de 55\%) y 18 con AR elevado exclusivo. La presencia de hipercloremia no se asoció con mejor estado de hidratación (porcentaje de déficit de peso en ambos grupos: $4,9 \% ; \mathrm{p}=0,81)$ ni con una respuesta terapéutica más rápida (con componente hiperclorémico: 9,5 horas; con AR elevado exclusivo: 11 horas; $\mathrm{p}=0,64)$.

Conclusiones. En niños con CAD, la prevalencia de AM con componente hiperclorémico fue del $55 \%$ y no se asoció con un mejor estado de hidratación ni con una salida más temprana de la descompensación metabólica.

Palabrasclave:cetoacidosisdiabética, hipercloremia, niño.

http:/ / dx.doi.org/10.5546/ aap.2018.e365 Texto completo en inglés:

http:/ / dx.doi.org/10.5546/ aap.2018.eng.e365

Cómo citar: Toledo I, Wainsztein R, Mannucci $\mathrm{C}$, et al. Impacto del componente hiperclorémico de la acidosis metabólica en el estado de hidratación y en el tratamiento de la cetoacidosis diabética. Arch Argent Pediatr 2018;116(3):e365-e370.

\section{INTRODUCCIÓN}

La cetoacidosis diabética (CAD) es una complicación grave de la diabetes mellitus (DM) tipo 1. En nuestro país, constituye la forma de presentación de la enfermedad en el $40 \%$ de los niños y es su principal causa de muerte (60-90\%), que, en la mayoría de los casos, se debe a edema cerebral asociado. ${ }^{1,2}$ Un signo cardinal que la caracteriza es la acidosis metabólica (AM) con anión restante (AR) elevado debido a la excesiva producción y retención de ácidos orgánicos o cetoácidos ( $\beta$-hidroxibutirato y acetoacetato) que titulan el bicarbonato plasmático y provocan el descenso de su concentración. ${ }^{3}$ Durante el tratamiento, la administración de insulina promueve la utilización de los ácidos cetónicos con regeneración de bicarbonato y la de fluidos endovenosos disminuye la producción de ácido láctico al mejorar el metabolismo aeróbico muscular. ${ }^{4}$ Sin embargo, al aumentar la diuresis, aumenta la excreción renal de aniones de los ácidos cetónicos que resultaban fuente de bicarbonato para la acción de la insulina. Esto, unido a que la reposición de electrolitos se realiza, en gran parte, con cloruro como anión, puede generar, en el curso de la evolución, un componente de acidosis hiperclorémica. ${ }^{4,5}$ Notablemente, en algunos pacientes que han mantenido un mejor estado de hidratación y, por lo tanto, una mayor excreción de ácidos cetónicos, puede encontrarse esta situación desde el ingreso. ${ }^{6}$ 
Además, pueden contribuir a la presencia de AM con componente hiperclorémico la pérdida de bicarbonato por orina (necrosis tubular aguda) o por materia fecal (gastroenteritis aguda), que provoca un incremento en la cloremia para mantener la electroneutralidad. ${ }^{6,7}$ Por consiguiente, dado que el componente hiperclorémico en la CAD previo al inicio del tratamiento podría reflejar un mejor estado de hidratación, sería esperable que su presencia al momento del diagnóstico se asociara con una mejor respuesta al tratamiento; sin embargo, según nuestro conocimiento, ningún estudio ha evaluado esta especulación. Los objetivos de este estudio fueron los siguientes: (1) determinar la prevalencia de AM con componente hiperclorémico previo a iniciar el tratamiento y (2) evaluar si dicho patrón metabólico se asociaba con mejor estado de hidratación y con menor tiempo de salida de la $\mathrm{CAD}$, en comparación con los pacientes con AM con AR elevado exclusivo.

\section{POBLACIÓN, MATERIAL Y MÉTODOS Pacientes}

Se realizó un estudio transversal y observacional, con recolección prospectiva de datos de pacientes menores de 18 años con CAD (ya fuera primera manifestación o con diagnóstico conocido) internados en el Hospital General de Niños Pedro de Elizalde entre el 1 de enero de 2014 y el 30 de junio de 2016. Se excluyeron aquellos con enfermedad renal crónica previa (que incluyó nefropatía diabética), aquellos que hubieran iniciado el tratamiento de esta complicación en otros centros de salud antes de ser derivados a nuestro Hospital y aquellos con sepsis o falla multiorgánica.

\section{Métodos}

Todos los pacientes que ingresaron en el estudio fueron tratados de acuerdo con la guía terapéutica de CAD de nuestro Hospital, ${ }^{3}$ la cual especifica las modificaciones por seguir en función de los distintos hallazgos clínicos y de laboratorio que pudieran surgir durante la evolución. Esto permitió cierta uniformidad en el tratamiento de una patología de manejo dinámico. Brevemente, los pacientes en shock o con $\mathrm{pH}<7,20$ fueron expandidos con $20 \mathrm{ml} / \mathrm{kg}$ de solución fisiológica y aquellos con $\mathrm{pH}<7,0$, con $10 \mathrm{ml} / \mathrm{kg}$ de bicarbonato de sodio $1 / 6$ molar $+10 \mathrm{ml} / \mathrm{kg}$ de solución fisiológica. Durante las primeras 24 horas, los pacientes fueron hidratados con $4000 \mathrm{ml} / \mathrm{m}^{2}$, que se pasaron en dos tiempos, la mitad en las primeras 6 horas (volumen: $2000 \mathrm{ml} / \mathrm{m}^{2}$; cloruro de sodio: $70 \mathrm{mEq} / \mathrm{L}$; cloruro de potasio: $30 \mathrm{mEq} / \mathrm{L}$; flujo de glucosa: $3,5 \mathrm{mg} / \mathrm{kg} / \mathrm{min}$ ) y la otra mitad en las 18 horas restantes (volumen: $2000 \mathrm{ml} / \mathrm{m}^{2}$; cloruro de sodio: $70 \mathrm{mEq} / \mathrm{L}$; cloruro de potasio: $40 \mathrm{mEq} / \mathrm{L}$; flujo de glucosa: $3,25 \mathrm{mg} / \mathrm{kg} / \mathrm{min}$ ). A su vez, se administró precozmente insulina corriente subcutánea $(0,1 \mathrm{U} / \mathrm{kg}$ o $0,2 \mathrm{U} / \mathrm{kg}$ si la glucemia era $<300 \mathrm{mg} / \mathrm{dl} \mathrm{o} \geq 300 \mathrm{mg} / \mathrm{dl}$, respectivamente) y se continuó con correcciones horarias de $0,1 \mathrm{U} / \mathrm{kg}$ hasta alcanzar las condiciones para pasar a control cada 4 horas (normohidratación, $\mathrm{pH} \geq 7,30$, bicarbonato $\geq 15 \mathrm{mEq} / \mathrm{L}$ y glucemia $\leq 250 \mathrm{mg} / \mathrm{dl})$. Se realizaron controles de laboratorio (urea, creatinina, estado ácido-base -EAB-, ionograma, glucemia, hemograma) al ingresar y luego a las 2, 6, 12 y 24 horas. Además, se controlaron en forma horaria los valores de glucemia, glucosuria y cetonuria con tiras reactivas y, en los casos de detectarse glucemia $\leq 250 \mathrm{mg} / \mathrm{dl}$, se realizó un análisis de laboratorio de sangre en dicho momento para verificar si el paciente se encontraba en condiciones metabólicas de pasar a controles cada 4 horas. A los fines de este estudio, dado que, cuando el paciente pasa a control cada 4 horas, se ha superado el período crítico de esta complicación, se consideró dicho momento como "salida de la CAD".

Se recabaron los siguientes datos: edad, género, si era primera manifestación o no de la enfermedad, síntomas acompañantes (fiebre, vómitos, diarrea, dolor abdominal), aplicación correcta o no de insulina. Al ingresar, antes de iniciar el tratamiento, se consignaron los datos clínicos (peso, frecuencia cardíaca, frecuencia respiratoria, estado neurológico según la escala de Glasgow ${ }^{8}$ diuresis y presión arterial media) y de laboratorio (hemograma, urea, creatinina, glucemia, sodio, potasio, cloro, $\mathrm{pH}$ plasmático, bicarbonato plasmático, dióxido de carbono y presencia de cetonuria y glucosuria). Posteriormente, se evaluaron los siguientes parámetros hasta la salida de la $C A D$ : tiempo en alcanzar dicha situación, volumen de líquido recibido (en $\mathrm{ml} / \mathrm{m}^{2}$ ), unidades de insulina administradas $(\mathrm{UI} / \mathrm{kg}$ ) y utilización de bicarbonato. Además, se consignó si el paciente requirió cuidados intensivos, si presentó edema cerebral y/o si falleció.

\section{Definiciones}

CAD: descompensación metabólica de la DM 
tipo 1 caracterizada por glucemia $>200 \mathrm{mg} / \mathrm{dl}$, $\mathrm{AM}(\mathrm{pH}<7,30 \mathrm{y} / \mathrm{o}$ bicarbonato $<15 \mathrm{mEq} / \mathrm{L})$, glucosuria y cetonuria. ${ }^{3}$

Sobre la base de los parámetros de laboratorio al ingresar, se definió lo siguiente:

- AM: concentración de bicarbonato plasmático $<22 \mathrm{mEq} / \mathrm{L}$ acompañado o no de $\mathrm{pH}<7,35$. 6,9

- AR: la diferencia entre sodio y la suma del cloro con el bicarbonato (valor normal: $12 \pm 2 \mathrm{mEq} / \mathrm{L}$ ). Su valor se ajustó según el $\mathrm{pH}$ plasmático (disminución de $1 \mathrm{mEq} / \mathrm{L}$ del AR por cada descenso de 0,1 de $\mathrm{pH}$ en sangre) y el primer valor disponible de albúmina sérica (disminución de $2 \mathrm{mEq} / \mathrm{L}$ del AR por cada descenso de $1 \mathrm{~g} /$ dl de albúmina plasmática). ${ }^{7}$

- Hipercloremia: cloro plasmático $>75 \%$ de la natremia. ${ }^{4,6}$

- $\mathrm{AM}$ con AR elevado: $\mathrm{AM}$ con $\mathrm{AR}>14 \mathrm{mEq} / \mathrm{L}$ con $\mathrm{pH}(7,35-7,45)$ y albúmina $(4-5 \mathrm{~g} / \mathrm{dl})$ normales en plasma. ${ }^{7,9}$

- AM con componente hiperclorémico: AM con AR elevado junto con la presencia de hipercloremia., ${ }^{4,6}$

- Osmolaridad: se calculó según la fórmula $\mathrm{Na}^{+}$plasmático $\times 2+$ glucemia $(\mathrm{mg} / \mathrm{dl}) / 18$ + uremia $(\mathrm{mg} / \mathrm{dl}) / 6$. Se consideró normal un valor de 275-290 mosm/ L. ${ }^{7}$

Edema cerebral: deterioro del nivel de conciencia acompañado por uno o más signos de hipertensión intracraneal (hipertensión arterial, bradicardia, alteraciones del patrón de respiración, alteraciones pupilares, estrabismo, visión borrosa, postura de descerebración o decorticación, paro respiratorio, evidencia radiológica o post mórtem de edema cerebral moderado o grave). ${ }^{2,10}$

Grado de deshidratación: se evaluó en función del porcentaje de déficit de peso, calculado como la diferencia entre el peso del alta y el registrado antes del inicio del tratamiento. ${ }^{11,12}$

Se consideró diuresis disminuida la eliminación de escasa cantidad de orina (ya fuera percibida por el paciente o referida por los padres) en las horas previas a la internación. ${ }^{12}$

Consideraciones éticas: El estudio fue aprobado por los Comités de Investigación y de Ética de nuestra Institución. Se obtuvo consentimiento informado de los padres o tutores y asentimiento de los niños mayores de 12 años.

\section{Análisis estadístico}

Se consideró como variable de resultado el tiempo en alcanzar la salida de la CAD y, como variable de predicción, la presencia de AM con componente hiperclorémico en el análisis de laboratorio de ingreso. En nuestro país, el tiempo mínimo en alcanzar la variable de resultado es de 8 horas, ${ }^{1}$ pero otros autores han comunicado un descenso de valores de glucemia $\leq 250 \mathrm{mg} /$ dl más rápidos (5,8 horas), es decir, aproximadamente, 2 horas menos. ${ }^{13}$ Sobre la base de esta información, se especula que, en los pacientes con hipercloremia, se podría alcanzar dicho objetivo: reducir en 2 horas el tiempo de salida. Para evidenciar esta diferencia en el tratamiento, se estimó un tamaño muestral (potencia de $80 \%$, nivel de confianza de 95\%) de 16 pacientes por grupo. Para el análisis, los pacientes fueron divididos en dos grupos: (1) AM con componente hiperclorémico y (2) AM exclusiva por AR elevado.

Las características clínicas y demográficas, así como los parámetros de laboratorio y evolutivos, fueron analizados descriptivamente. Las variables cuantitativas presentaron distribución no paramétrica (test de Shapiro-Wilk), por lo que se expresaron como mediana (intervalo) y fueron comparadas con el test de Wilcoxon. Las variables categóricas se expresaron como frecuencia de presentación y porcentaje, y se compararon con el test de chi cuadrado o con el test exacto de Fisher según correspondiera. Se utilizó el programa Statistix ver. 7 (IBM version; Analytical Software, Tallahassee, FL) y se consideró un valor de $p<0,05$ (a 2 colas) como estadísticamente significativo.

\section{RESULTADOS}

Durante el período de reclutamiento, se internaron, en nuestra institución, 52 pacientes con CAD. Doce de ellos fueron excluidos: 7 por haber recibido tratamiento previo al análisis de laboratorio inicial, 4 por haber sido derivados de otro hospital y 1 porque presentó falla multiorgánica. Por consiguiente, la muestra de estudio quedó conformada por 40 pacientes (23 mujeres) con una mediana de edad de 14,5 años (2,4-18); 5 correspondieron a primera manifestación de la enfermedad.

En 22 pacientes, se observó AM con componente hiperclorémico en el inicio (prevalencia de 55\%) y, en 18 casos, AM exclusiva por AR elevado. Ambos grupos de pacientes fueron similares en las características demográficas, de laboratorio y evolutivas, excepto en la prevalencia de dolor abdominal, que fue significativamente mayor en los pacientes sin hipercloremia (Tablas 1-3). De manera remarcable, 
la presencia de hipercloremia al ingresar no se asoció con un mejor estado de hidratación (la mediana del porcentaje de déficit de peso tanto en pacientes con componente hiperclorémico como en pacientes con AR elevado exclusivo fue de $4,9 \%, p=0,81$ ) ni con una mejor respuesta terapéutica. En efecto, los pacientes con componente hiperclorémico alcanzaron la fase

TABLA 1. Comparación de variables demográficas y clínicas al ingresar entre pacientes con cetoacidosis diabética, con acidosis metabólica, con componente hiperclorémico y con anión restante elevado exclusivo

\begin{tabular}{lccc}
\hline Variable & $\begin{array}{c}\text { AM con componente } \\
\text { hiperclorémico (n= 22) }\end{array}$ & $\begin{array}{c}\text { AM con AR elevado } \\
\text { exclusivo (n= 18) }\end{array}$ & Valor de $\boldsymbol{p}$ \\
\hline Edad (años) & $15,5(5,5-18)$ & $13,25(2,4-18)$ & 0,45 \\
Sexo (F/M) & $13 / 9$ & $10 / 8$ & 0,82 \\
Peso inicial (kg) & $44(15,5-67)$ & $42(12-64)$ & 0,79 \\
Primera manifestación (sí/no) & $2 / 20$ & $3 / 15$ & 0,65 \\
Fiebre & 3 & 2 & 1 \\
Vómitos & 18 & 12 & 0,46 \\
Diarrea & 2 & 0 & 0,49 \\
Dolor abdominal & 13 & 17 & 0,02 \\
Diuresis disminuida & 5 & 4 & 0,68 \\
Presión arterial media (mmHg) & $90(85-95)$ & $85(70-95)$ & 0,58 \\
Escala de Glasgow & $15(13-15)$ & $15(14-15)$ & 0,56 \\
Aplicación correcta de insulina & $7^{*}$ & $3^{\#}$ & 0,45 \\
Frecuencia cardíaca (lpm) & $120(88-140)$ & $120(88-150)$ & 0,69 \\
Frecuencia respiratoria (rpm) & $22(14-30)$ & $24(16-45)$ & 0,6 \\
\hline AMtacidosis matrón
\end{tabular}

AM: acidosis metabólica; AR: anión restante; F/ M: femenino/masculino; lpm: latidos por minuto; rpm: respiraciones por minuto; ${ }^{*} \mathrm{n}$ total $=20 \mathrm{y}{ }^{\#} \mathrm{n}$ total $=15$ (debido a que se excluyeron los pacientes con la primera manifestación de su diabetes).

TABLA 2. Comparación de parámetros de laboratorio entre pacientes con cetoacidosis diabética, con acidosis metabólica, con componente hiperclorémico y con anión restante elevado exclusivo

\begin{tabular}{lccc}
\hline Parámetro de laboratorio & $\begin{array}{c}\text { AM con componente } \\
\text { hiperclorémico (n= 22) }\end{array}$ & $\begin{array}{c}\text { AM con AR elevado } \\
\text { exclusivo (n= 18) }\end{array}$ & Valor de $p$ \\
\hline $\mathrm{pH}$ & $7,19(6,94-7,29)$ & $7,15(7,06-7,28)$ & 1 \\
Dióxido de carbono (mmHg) & $23(13-37)$ & $26(18-40)$ & 0,09 \\
Bicarbonato (mEq/L) & $9(4,1-14,3)$ & $10(5,8-14)$ & 0,22 \\
Sodio (mEq/L) & $140,5(133-149)$ & $143(135-156)$ & 0,08 \\
Potasio (mEq/L) & $4,4(2,8-6,4)$ & $4,5(3,2-6,8)$ & 0,53 \\
Cloro (mEq/L) & $111(106-118)$ & $105(97-113)$ & 0,0001 \\
Glucemia (mg/dl) & $435(214-1077)$ & $431,5(205-847)$ & 0,85 \\
Creatinina (mg/dl) & $0,5(0,4-0,9)$ & $0,69(0,5-0,8)$ & 0,46 \\
Urea $(\mathrm{mg} / \mathrm{dl})$ & $32(18,8-58)$ & $41(20-114)$ & 0,16 \\
Leucocitos $\left.(\mathrm{mm})^{3}\right)$ & $13800(6200-36500)$ & $13500(7500-23500)$ & 0,97 \\
Hematocrito (\%) & $45(38,7-50,8)$ & $44(34-49)$ & 0,25
\end{tabular}

AM: acidosis metabólica; AR: anión restante.

TABLA 3. Comparación del tratamiento recibido y de la evolución entre pacientes con cetoacidosis diabética, con acidosis metabólica, con componente hiperclorémico y con anión restante elevado exclusivo

\begin{tabular}{lccc}
\hline Variable & $\begin{array}{c}\text { AM con componente } \\
\text { hiperclorémico }(\mathbf{n = 2 2})\end{array}$ & $\begin{array}{c}\text { AM con AR elevado } \\
\text { exclusivo (n= 18) }\end{array}$ & Valor de $p$ \\
\hline Déficit de peso (\%) & $4,9(3,3-8,9)$ & $4,9(3-10,9)$ & 0,81 \\
Volumen total de líquido infundido $\left(\mathrm{ml} / \mathrm{m}^{2}\right)$ & $3125(1400-5300)$ & $3110(1500-4500)$ & 0,49 \\
Insulina total aplicada (UI/ kg) & $0,95(0,3-3,4)$ & $1(0,5-1,9)$ & 0,72 \\
Uso de bicarbonato de sodio & 3 & 1 & 0,61 \\
Tiempo de salida de la CAD (horas) & $9,5(3,3-24)$ & $11(5,75-20)$ & 0,64 \\
Edema cerebral & 0 & 0 & 0 \\
Requerimiento de cuidados intensivos & 0 & 0 & 0 \\
Muerte & 0 & 0 & 0 \\
\hline
\end{tabular}

AM: acidosis metabólica; AR: anión restante; CAD: cetoacidosis diabética. 
de salida luego de 9,5 horas y los restantes, a las 11 horas $(p=0,64)$, con similares requerimientos de fluidos endovenosos y de insulina subcutánea (Tabla 3).

Ningún paciente presentó edema cerebral ni requirió cuidados intensivos. Tampoco se registró ningún fallecimiento.

\section{DISCUSIÓN}

En la reposición de electrolitos durante la CAD, se emplea un aporte generoso de cloruro (como anión), por lo que el desarrollo de hipercloremia durante el tratamiento es un hallazgo habitual en estos pacientes (58$94 \%){ }^{14-18}$ Sin embargo, existe escasa información sobre la presencia de hipercloremia al momento del ingreso. ${ }^{14,15}$ En este estudio, se analizó a 40 niños internados por CAD durante un período de 2 años y medio. La mediana de edad del grupo estudiado fue de 14,5 años y hubo un leve predominio de mujeres. Merece mencionarse que solo $5(12,5 \%)$ correspondían a primera manifestación de la enfermedad, una proporción menor a la comunicada en otros estudios. ${ }^{1}$ Esto podría deberse a que el Servicio de Nutrición y Diabetes de nuestro Hospital posee en seguimiento un gran número de pacientes, los cuales, habitualmente, son también asistidos en nuestro centro en presencia de complicaciones.

El análisis del patrón metabólico al momento del ingreso hospitalario de nuestros pacientes, antes de recibir cualquier tipo de tratamiento, reveló una prevalencia de componente hiperclóremico de 55\%, hallazgo que resultó marcadamente elevado con respecto a lo comunicado en estudios previos. Mrozik y cols., en una serie de 59 niños con CAD, no encontraron hipercloremia en el inicio del cuadro; sin embargo, debe tenerse en cuenta que consideraron hiperclorémicos a aquellos niños que carecían de AR elevado (AR > 16) ${ }^{14}$ En tanto que Taylor et al., definiendo hipercloremia como la relación plasmática de cloro/sodio $>0,79$, observaron, en una serie de 18 niños, una prevalencia del $6 \% .{ }^{15}$ Una posible causa de las diferencias de prevalencia encontradas entre el presente y los mencionados estudios podría ser la definición utilizada de hipercloremia, que, en nuestro caso, fue la más aceptada en la bibliografía (relación plasmática de cloro/sodio $>0,75$ ). ${ }^{4,6} \mathrm{Si}$ se hubiera empleado la definición de Taylor, nuestra prevalencia de hipercloremia hubiera descendido a la mitad, un porcentaje que aún continúa siendo mayor que en los otros estudios.
Como ya fue mencionado, cabría esperar que los pacientes con componente hiperclorémico se encontraran mejor hidratados y que, consecuentemente, resolvieran el cuadro metabólico con más rapidez; sin embargo, ningún estudio, según nuestro conocimiento, analizó este postulado en el escenario clínico. En nuestra serie, no se mostraron diferencias en el estado de hidratación observadas por el porcentaje de déficit de peso al momento del ingreso. De acuerdo con este hallazgo, tampoco se encontraron diferencias en ciertos parámetros de laboratorio modificables por el volumen del líquido extracelular, como hematocrito y urea (Tabla 2), ni tampoco con parámetros clínicos asociados a mayor déficit previo (Tabla 1). De manera esperable, ya que no hubo diferencias en el estado de hidratación, la respuesta al tratamiento medida en función del tiempo de salida de la $C A D$ entre ambos grupos también fue comparable (Tabla 3).

Una limitación que debe mencionarse es que la presencia de pérdidas previas al ingreso (vómitos, diarrea, hipertermia) fue evaluada en forma cualitativa y se desconoció su magnitud. Más allá de esto, parece difícil realizar un estudio que pueda evaluar estas variables con precisión, ya que estos eventos ocurren antes de la internación; además, el estado de hidratación en nuestros pacientes fue evaluado en función del porcentaje de déficit de peso, que era una medida cuantificable y objetiva. Asimismo, si bien la guía de tratamiento asegura el control horario para captar rápidamente la salida de la $\mathrm{CAD}$, no es posible descartar con certeza pequeños desfasajes horarios en el momento de realizar los controles.

Como fortaleza de este estudio, debe destacarse que la recolección de datos se realizó en forma prospectiva y que todos los pacientes fueron tratados con el mismo esquema terapéutico.

\section{CONCLUSIÓN}

En nuestros pacientes con CAD, la prevalencia de AM con componente hiperclorémico fue del $55 \%$ y su presencia no se asoció con un mejor estado de hidratación ni con una recuperación más temprana de la descompensación metabólica.

\section{REFERENCIAS}

1. Ferraro M,Ozuna B, RamosO, etal. Estudio multicéntrico de cetoacidosis diabética en servicios pediátricos de Argentina. Rev Soc Argent Diabetes 2009;43:127-36.

2. Glaser N, BarnettP, McCaslin I, et al. Risk factors for cerebral edema in children with diabetic ketoacidosis. The Pediatric 
Emergency Medicine Collaborative Research Committee of the American Academy of Pediatrics. $N$ Engl J Med 2001;344(4):264-9.

3. Ramos O, Ferraro M, BarbeitoS. Cetoacidosis diabética. En Voyer L, ed. Criterios de diagnóstico y tratamientoen Pediatría. 2. ${ }^{\text {a }}$ ed. Buenos Aires: Journal; 2006.Págs.95-100.

4. Repetto HA. Desequilibrios hidroelectrolíticos y ácidobásicos en la descompensación de la diabetes. Arch Argent Pediatr 2000;98(1):47-50.

5. Adrogué HJ, Eknoyan G, Suki WK. Diabetic ketoacidosis: role of the kidney in the acid-basehomeostasis re-evaluated. Kidney Int 1984;25(4):591-8.

6. Massó D, Repetto HA. Fisiología y fisiopatología de la homeostasis del estado ácido base. En: Nefrología pediátrica. 2. ${ }^{\text {da }}$ ed. Buenos Aires: FUNDASAP; 2008.Págs.355-80.

7. Rose B, Post T. Acidosis Metabólica. En Rose BD, Post TW, eds. Trastornos de los electrolitos y del equilibrio ácido base. $5^{\mathrm{a}}$ ed. Boston: Marban; 2002.Págs.78-646.

8. Moore L, Lavoie A, Camden S, et al. Statistical Validation of the Glasgow Coma Score. J Trauma 2006;60(6):1238-44.

9. Tejedor A, Caramelo C. Acidosis metabólica. En Ayus JC, Caramelo C, eds. Agua, electrolitos y equilibrio ácido-base: aprendizaje mediante casos clínicos. Madrid: Panamericana; 2006.Págs.188-222.

10. Bohn D. Understanding the pathophysiology of cerebral edema in diabetic ketoacidosis: another brick in the wall? Pediatr Crit Care Med 2010;11(3):421-3.

11. Freedman SB, Vandermeer B, Milne A, et al. Diagnosing clinically significant dehydration in children with acute gastroenteritis using noninvasive methods: a meta-analysis. J Pediatr 2015;166(4):908-916.e1-6.

12. PringleK, ShahSP, Umulisa I, et al. Comparing the accuracy of the three popular clinical dehydration scales in children with diarrhea. Int J Emerg Med 2011;4:58.

13. Ballesteros Calderón AL, Meneses Muñoz AP, Ríos Patiño D, et al. Características epidemiológicas y clínicas y hallazgos de laboratorio de los niños menores de 15 años con cetoacidosis diabética atendidos en el Hospital UniversitarioSan Vicente Fundación en Medellín. IATREIA 2013;26(3):278-290.

14. Mrozik LT, Yung M. Hyperchloraemic metabolic acidosis slows recovery in children with diabetic ketoacidosis: a retrospective audit. Aust Crit Care 2009;22(4):172-7.

15. Taylor D, Durward A, Tibby S, et al. The influence of hyperchloraemia on acid base interpretation in diabetic ketoacidosis. Intensive Care Med 2006;32(2):295-301.

16. Basnet S, Venepalli PK, Andoh J, et al. Effect of normal saline and half normal saline on serum electrolytes during recovery phase of diabetic ketoacidosis. J Intensive Care Med 2014;29(1):38-42.

17. Orlowsky JP, Cramer CL, Fiallos MR. Diabetic Ketocidosis in the pediatric ICU. Pediatr Clin North Am 2008;55(3):577-87.

18. Ferreira JP, Hamui M, Torrens M, et al. The Influence of Chloride for the Interpretation of Plasma Bicarbonate During the Treatment of Diabetic Ketoacidosis. Pediatr Emerg Care 2017 [Epub ahead of print]. 